\title{
Tools for Assessment of Occupational Health Risks of some Engineered Nanoparticles and Carbon Materials Used in Semiconductor Applications
}

\author{
Ponnapat Watjanatepin and Dimiter Prodanov
}

Additional information is available at the end of the chapter

http://dx.doi.org/10.5772/intechopen.76567

\begin{abstract}
Engineered nanomaterials (ENM) are used in a wide variety of applications: from cosmetics and paints to sportswear and semiconductor chips. While for chemicals there are established regulatory frameworks dealing with the risk for the consumers, workers, and the environment, this is not the case for nanomaterials. This is precisely why ENMs are used-the properties of matter change at the nanoscale and become dependent on the particle morphology and size. Our understanding on how such nano-systems react with biological matter, such as cells and tissues, is far from complete, and this brings about an increasing level of uncertainty in the research and development process. This chapter will give an overview of several materials, which are either used or have potential applications in nanoelectronics. While silicon dioxide and metal oxide nanoparticles are used in semiconductor processing in standard polishing steps, applications of carbon materials may be more disruptive. As promising materials with broad applications, we focus on carbon nanotubes and graphene. So-identified materials are used to illustrate the use of different risk assessment tools in the occupational setting of nanoelectronics. The application of such tools in itself is also a growing area of research efforts supported by international stakeholders, such as the European Commission.
\end{abstract}

Keywords: nanosafety, nanomaterials, nanotechnology, semiconductor

\section{Introduction}

An increasing number of engineered nanomaterials (ENM) enter the market in everyday products spanning from healthcare and leisure to electronics, cosmetics, energy, agriculture, food, and transport. By 2014, there have been more than 1600 nanotechnology-enabled products in 


\begin{tabular}{|c|c|c|}
\hline Sector & Applications/products & Nanomaterial \\
\hline \multirow[t]{4}{*}{ Electronics } & Electrically conductive polymers & CNTs \\
\hline & Abrasive particles in CMP processes & $\mathrm{SiO}_{2}, \mathrm{CeO}_{2}, \mathrm{Al}_{2} \mathrm{O}_{3}$ \\
\hline & Sensors & CNTs, graphenes \\
\hline & Supercapacitors & CNTs, graphenes \\
\hline \multirow[t]{3}{*}{ Energy } & Electrodes in energy conversion and storage & CNTs, graphenes, other metallic NPs \\
\hline & Batteries & $\mathrm{Al}_{2} \mathrm{O}_{3}$ \\
\hline & Fuel cells & Graphenes \\
\hline Textiles & Nanofibers and antibacterial textiles & Nano-Ag \\
\hline \multirow[t]{3}{*}{ Construction } & Insulation & Silica-aerogel \\
\hline & Light transmission & Silica-aerogel \\
\hline & Dirt-repellent paints & $\mathrm{SiO}_{2}$ \\
\hline \multirow[t]{4}{*}{ Medicine } & Drug carriers & $\mathrm{SiO}_{2}, \mathrm{CeO}_{2}$ \\
\hline & Biomedical imaging & $\mathrm{SiO}_{2}$ \\
\hline & Dental implants & $\mathrm{Al}_{2} \mathrm{O}_{3}, \mathrm{SiO}_{2}$ \\
\hline & Biosensors & CNTs, graphenes \\
\hline \multirow[t]{5}{*}{ Automotive } & Hydrogen storage & MOFs \\
\hline & Antifog coating for windshield/mirror & $\mathrm{TiO}_{2}$ \\
\hline & Scratch proof lacquer & Nanoceramics \\
\hline & Fuel additives & $\mathrm{CeO}_{2}$ \\
\hline & Catalysis & $\mathrm{SiO}_{2}$ \\
\hline Cosmetics & Sun screen & $\mathrm{TiO}_{2}$ \\
\hline \multirow[t]{5}{*}{ Various } & Nanofillers & CNTs, graphenes \\
\hline & Nanocomposites & $\mathrm{CNT}$ /polymer matrix, CNT/ceramic matrix \\
\hline & Filters & CNTs \\
\hline & Anticorrosive coatings & Graphenes \\
\hline & Nanofilms & \\
\hline
\end{tabular}

Sources: Refs. [2-5].

Table 1. Leading industrial applications of common engineered nanomaterials.

commerce [1]. A summary of ENM applications is given in Table 1. While there are natural sources of nanoparticles, such as volcanoes, forest fires, or sea storms, the engineered nanomaterials are purposefully made to achieve certain prescribed functionality. Such desirable characteristics can include increased strength of the material, its chemical reactivity, or altered electrical properties. The terms ENM and nanomaterials will be used interchangeably throughout the chapter.

Nanomaterials can be classified broadly as particles, which have oval shapes; fibers, which are tube-like but may exhibit complex branching; and sheets, which are film-like but again may 
exhibit branching or defects. Interested readers are directed to Buzea et al., for a review [6]. The European Commission defines nanomaterials as "natural, incidental or manufactured material containing particles, in an unbound or as an aggregate or agglomerate and where $50 \%$ or more in the number size distribution, one or more dimensions is in the range of 1-100 nm."

The size cutoff in common definitions is somehow arbitrary. It, however, conveys the understanding that properties of the material features at the nanoscale (i.e., the so-called nanoforms of materials) can substantially differ from the properties of materials in bulk. This can be explained by two main factors affecting the physicochemical properties because of their small sizessurface effects and quantum effects [7]. The surface effects occur due to the fact that as the size of nanoparticle decreases, the surface area relative to the volume increases as the inverse power of the diameter $\sim 1 / d$. The relative increase in area provides a greater overall surface per unit mass available for reactions as well as higher charge and energy densities, which influence the surface chemistry of ENM (reviews in [6, 8]). Quantum effects result from the confinement of electrons when the particle radius decreases [6]. Electron confinement in turn influences the capability for electrons to be donated or accepted, hence influencing their electrical charge distribution and the catalytic capability.

This chapter aims to familiarize readers with nanomaterials, which are either used in the semiconductor manufacturing or have current and promising future applications in electronics industries. Due to the limited volume, we have focused on silica, certain metallic nanoparticles, and carbon materials, such as carbon nanotubes and graphene. The applications of these ENMs will be discussed in Section 2. At the same time, the use of ENMs raises questions about potential unintended risks for the workers, consumers, or environment. Consequently, an overview of the nanotoxicological studies for all these three classes of nanomaterials is presented in Section 3. Since the manipulation and use of ENMs warrant for safety precautions, different relevant control banding tools for risk assessment will be summarized in Section 4. Finally, the concept of tiered risk assessment approach is introduced in Section 4.5 as a flexible framework, which is able to decrease uncertainty as new information becomes gradually available.

\section{Applications}

\subsection{Nanoelectronics as a case study}

Nanoelectronics relies on multiple semiconductor processes resulting in pattering of macroscopic objects (silicon wafers) on the nanoscale. Overall, the semiconductor industry can be considered predominantly as a downstream user of nanomaterials. Nanoelectronics enjoys a very fast innovation cycle governed by Moore's law. This brings about a variety of new materials and combinations into production, while some of them are in nanoform. As a result, new materials, including a variety of nanoparticles, have been introduced in the development of advanced technology nodes predominantly in polishing operations. Engineered nanomaterials are used in several processing steps, but they can also be generated as side products of several generic processes. 
The background of materials studies in the field of occupational health naturally focuses on the release, monitoring, mitigation of exposure, and health implications. The relevance of the work for the semiconductor industry is in those studies proven by naming few examples for the application of nanomaterials in semiconductor technology [9]. The semiconductor industry can be taken as a use case on how potential occupational and environmental risks brought about by nano-enabled products are governed. Specifics of this industry, which make it interesting, are several:

- Semiconductor mass manufacturing employs top-down high-precision approaches, which are highly standardized.

- Nanoelectronics enjoy a very fast innovation cycle.

- Due to the fast innovation cycle, there is a safety culture used to dealing with uncertainties.

An example of such standardized process is the complementary metal-oxide-semiconductor (CMOS) fabrication, which is the focus of the safety assessment undergoing in the H2020 project NanoStreeM [4]. The project findings demonstrate that chemical mechanical planarization (CMP) is the most common standard process, where ENMs are used. It should be noted that wafer-scale integration of carbon nanomaterials is not yet industrially available.

\subsection{Metal oxides NPs and silica}

Semiconductor industry uses nanoparticles in several processing steps, particularly in CMP, which is a standardized processing step during the manufacturing of semiconductors, for example, the CMOS processing. The purpose of CMP is to achieve a high degree of planarity for the wafer surface and remove the excess of deposited material. The importance of having very flat surface is due to the fact that even very slight undulations, or small defects and scratches on the wafer surface, would degrade the yield from a wafer and thus result in an economic loss $[5,10,11]$.

The planarization process employs a mechanical force to synergistically work with the chemical reactions to remove metallic surfaces in order to achieve planarity for the wafers [12]. During CMP processing, the wafer is loaded onto the wafer carrier. Then the slurry emulsion is released from the dispersing head and reacts with the wafer surface so that chemical reactions help create chemically modified surface. Simultaneously, slight force is applied downward so that friction force develops between the wafer carrier, polishing pad and the abrasives in the slurry. This results in removal of deposited materials. Additionally, CMP tools also require pad conditioning. This conditioning is needed in order to maintain a stable rate of material removal. These pad conditioners are usually disks with diamond particles that will help stabilize the surface roughness of the pad [10].

Besides nanoparticle abrasives different slurries are composed of proprietary components, which optimize the desired functionalities. The roles of each component can be summarized in general as [5, 12]:

- Molecules for $\mathrm{pH}$ adjustments maintain stable $\mathrm{pH}$ for the slurry.

- Complexing agents aid in the solubilization of the dissolved metallic components. 
- Oxidizers improve the selectivity of the slurry by promoting dissolution or protecting against the removal of a certain surface component.

- Corrosion inhibitors stop the corrosive effects on the wafer surfaces.

- Biocides inhibit growth of biological organisms.

- Abrasive particles increase the frictional force needed to remove the chemically modified surfaces during the CMP process.

The abrasive particles are nanoparticles in the range of 20-100 nm [10]. An important point for the use of nanoparticles in the CMP slurry is that the nanoparticles tend to aggregate or agglomerate into bigger sizes. Agglomeration and aggregation of particles can cause $\mathrm{pH}$ variations at different areas of the slurry. This in turn causes nonuniformity on the wafer surfaces after the polishing process [11]. To avoid the agglomeration or aggregation of the abrasive nanoparticles, CMP slurries are often mixed on site prior to the use via a CMP slurry mixing and distribution system. Alternatively, coating of the abrasive nanoparticles can also be used to maintain stability. Currently, the most widely used nanoparticles acting as abrasives in CMP slurries are silica (silicon dioxide), alumina (aluminum oxide), and cerium oxide [5].

\subsection{Carbon nanotubes}

Carbon nanotubes (CNTs) were discovered in 1991 and since then have found many applications $[13,14]$. There are three main categories of CNTs based on the wall structure: singlewalled carbon nanotubes (SWCNTs), double-walled carbon nanotubes (DWCNTs), and multiwalled carbon nanotubes (MWCNTs) (overview in [13]). The main structural difference between MWCNTs and the SWCNTs is that MWCNTs contain multiple concentric carbon sheets [14]. CNTs possess many unique physicochemical properties that supersede other carbon-based materials used formerly, such as graphite, diamond, and fullerenes. The main physicochemical properties of CNTs are summarized in Table 2.

In the field of energy conversion and storage, CNTs are mostly applied in mainly two applications: electrodes in lithium-ion batteries and supercapacitors [13, 14]. The use of CNTs provides high surface area. CNTs, especially SWCNTs, are good candidates for use as electrodes for Li-ion batteries. It was shown that SWCNTs exhibit good intercalation of Li-ions on their walls as well as have higher interstitial density than the traditionally used graphite electrodes [15]. Additionally, the incorporation of CNTs as electrodes for Li-ion batteries has a simpler fabrication process than those of the conventional electrodes [16]. This is because layers of metal mixture substrates and electrical conductor must be deposited on the conventional electrodes, while CNTs can be directly grown onto the electrodes themselves. However, the potential discharge of the CNT electrodes is lower than those of the conventional types [14]. Beyond the use with Li-ion batteries, CNTs are also depicted and studied as candidate material for supercapacitors.

Supercapacitors are considered as a high-performing alternative for Li-ion batteries. The energy storage properties of supercapacitors are highly dependent on the surface area since the energy is stored in a charged double layer that is formed after the application of a voltage source. Hence, CNTs, having high surface-area-to-volume ratio, are especially suitable [17]. 


\begin{tabular}{llll}
\hline Property (unit) & SWCNTs & MWCNTs & Graphenes \\
\hline Tensile strength $(\mathrm{GPa})$ & $50-500$ & $10-60$ & $>1000$ \\
Elastic modulus (TPa) & $\approx 1$ & $0.3-1$ & 1 \\
Electrical conductivity $(\mathrm{S} / \mathrm{m})$ & $1.3-1.5$ & $1.8-2.0$ & 2000 \\
Specific surface area $\left(\mathrm{m}^{2} / \mathrm{g}\right.$ by BET) & $>1000$ & $>500$ & 2630 \\
\hline
\end{tabular}

Source: Ref. [3].

Table 2. Properties of SWCNTs, MWCNTs, and graphenes.

CNTs are also widely used in the field of sensors due to their unique properties like the high surface area, electrical conductivity and adsorptive properties. These properties contribute to a simpler fabrication of the CNT-based sensor with lower response time and higher sensitivity. The easier fabrication steps also led to easier incorporation into various electronic circuits, miniaturization of the component, and lower production cost and energy consumption [14].

CNTs could be functionalized with metals, metal oxides, polymers, or biomacromolecules [3]. Although CNTs are promising candidate materials for use in electronics industry, the synthesis and purification of CNTs are two of the biggest concerns. The syntheses of CNTs are mostly conducted either via chemical vapor deposition, arc discharge, or laser ablation [14, 17]. All of these processes require metal catalysts during the synthesis. These metallic catalysts left residual particles on the CNT surfaces which decrease the purity of the obtained CNTs. Impurities or changes in surface composition can affect the surface effect of the nanomaterial, which in turn modifies the physicochemical properties of the aforementioned nanomaterial. Subsequently, their environmental fate, uptake, and toxicological properties will also be affected and can differ from other nanomaterials of the same kind. Many purification steps involving liquid/gas phases as well as oxidation reactions were proposed, but these methods affect the physicochemical properties of the CNTs accordingly $[18,19]$.

\subsection{Graphene}

Graphene contains $s p^{2}$ planar carbon sheet structure. Due to this two-dimensional structure, graphene is one of the thinnest yet mechanically strongest nanomaterials available [20, 21]. Additionally, graphenes also possess excellent optical properties as well as high mobility of electrons. The physicochemical properties of graphene are summarized in Table 2. When referring to graphene-based materials, the other two derivatives of pristine graphene are also included: graphene oxide (GO) and reduced graphene oxide (rGO).

When referring to the field of energy storage and conversion, graphenes are mostly used in Li-ion batteries as well as in optoelectronic devices, such as solar cells [3, 22, 23]. In Li-ion batteries, graphene-based nanomaterials, specifically, graphene oxides (GO), are mostly used as buffers to prolong the storage lifespan. The unique tensile strength and electrical conductivity of graphene-based nanomaterials protect the electrodes from being pulverized and facilitate the charge-discharge cycles of the batteries [20]. In the applications with solar cells, graphene-based nanomaterials are functionalized with quantum dots (QDs) to form a hybrid matrix that raises the performance of a photovoltaic array [20, 22]. In contrast to CNTs, graphene-based materials 
are more flexible due to their mechanical properties. The use of graphene-based materials for sensors is increasing gradually. Owing to their extremely high available surface-area-to-volume ratio and electrical conductivity, graphene-based sensors have a performance on par or even higher than those of CNTs or Si-based sensors [20].

In contrast to CNTs, the problems of impurities faced during the synthesis of graphenes are much less. However, the degree of reproducibility during the fabrication of graphene-nanoparticle matrix is a topic of concern [3,20,21]. It is particularly complicated to obtain uniformly dispersed nanoparticles on these graphene sheets. Hence, the nonuniformity can and will affect the physicochemical properties of these graphenes and eventually hinder the industrial use of graphenes. Therefore, additional studies on the industrial applications of graphene-based nanomaterials are still needed to overcome this drawback and exploit the potential of the material in the electronics industry.

\section{Nanotoxicology}

\subsection{Nanotoxicology of silica}

Silica ENM is used in slurry formulations. Their density is 2.196 (amorphous) $\mathrm{g} / \mathrm{cm}^{3}$ [24]. Recent publications have demonstrated that silica NPs can become cytotoxic both in vitro and in vivo in a dose-, size-, and cell-type-dependent manner [25-27]. The most common route of exposure for silica NPs is via inhalation [28-30]. On a second place is the ingestion exposure route.

In vitro studies have demonstrated that silica NP toxicity is mostly caused by the production of reactive oxidative species (ROS) [31-35]. The surface silica radical groups can generate free radicals and ROS by the Fenton reactions. In particular, ROS can be generated by the failed phagocytosis of silica NPs. The latter mechanism is valid for all cases of incomplete phagocytosis of NPs; thus, it is also applicable for alumina and cerium oxide. Fubini, Hubbard, and Lehman demonstrated that the fractured silica surfaces, which are more prone to release free silanol radicals, can initiate Fenton reactions in water $[35,36]$ :

$$
\begin{gathered}
\bullet \mathrm{O}^{-}+2 \mathrm{H}^{+}+\mathrm{e}^{-} \rightarrow \mathrm{H}_{2} \mathrm{O}_{2} \\
-\mathrm{SiOO}^{\bullet}+\mathrm{H}_{2} \mathrm{O}_{2} \rightarrow-\mathrm{SiOH}+\mathrm{HO}^{\bullet}+\mathrm{O}_{2} \\
-\mathrm{SiO}^{\bullet}+\mathrm{H}_{2} \mathrm{O} \rightarrow-\mathrm{SiOH}+\mathrm{HO}^{-}
\end{gathered}
$$

which are catalyzed by the $\mathrm{Fe}$ oxidation as $\mathrm{Fe}^{2+}+\mathrm{O}_{2} \rightarrow \mathrm{Fe}^{3+}+\cdot{ }^{\bullet} \mathrm{O}_{2}^{-}$[33]. The hydroxyl radicals can increase cytotoxicity by increasing oxidative stress (e.g., the intracellular concentration of hydrogen peroxide). It was also shown that when undergoing incomplete phagocytosis, cells tend to produce ROS and increase the level of intracellular oxidative stress [31]. Subsequently, this leads to an increase in cytotoxicity and damage to various cellular compartments. Excessive intracellular ROS concentration has been reported to contribute to higher cytotoxicity by directly increasing cellular stress and by indirectly damaging the mitochondria and macromolecules which can further lead to genetic damage, carcinogenesis, or reproductive defects [8, 37]. 
As noted previously, as the particle size decreases, there is an increase in the surface area fraction which influences the surface chemistry. Moreover, the small size of the NPs helps them to evade clearance mechanisms in the body; therefore, the retention time is further increased, rendering them more biopersistent [7]. These factors contribute to a increased potential for reactivity, resulting in higher ROS production, lipid peroxidation, and the damage to cell membranes [38-40]. This can ultimately lead to apoptotic cell death.

Several in vitro studies emulating ingestion as a route of exposure by using cell lines from the gastrointestinal tract have been conducted. According to a recent review, in vitro studies of immunotoxicity were conducted mainly on monocytes and macrophages which showed inflammatory responses more severe than exposure to silica in the micron range [25]. However, this result is not representative of the entire immune system; hence, further studies are still needed before firm conclusions can be drawn.

The 2016 Organization for Economic Co-operation and Development (OECD) dossier on silicon dioxide suggested that amorphous nanosilica seems not to exhibit carcinogenic, mutagenic, and reprotoxic (CMR) properties [41]. This is in accordance with other studies showing no genotoxic effects of silica NPs [42].

In vivo studies with nanosilica were mostly done to study inhalation exposure. The numbers are significantly less when compared to in vitro studies, and no chronic exposure study was found. The results reported in literature are conflicting due to different dosages, sizes, and animal species used. At present, it is still not possible to draw definite conclusions from the in vivo studies due to their limited number. Moreover, most of these studies do not follow a standardized operating procedures, and therefore, it is not possible to compare the results or difficult to interpret the results [43]. Readers are directed to the recent review of Murugadoss et al. for more information [25]. Different studies have shown that silica NPs can induce cytotoxicity by forming ROS due to their small sizes resulting in increased reactivity. Although ROS formation has also been linked to CMR properties, no CMR effects were found after exposure to silica NPs. In addition, the studies have shown that silica NPs are capable of forming protein corona which influences the effective concentration used in toxicological studies. This further puts an emphasis on the importance of the appropriate dosage/concentration selection for in vitro and in vivo studies as the NPs could interact with the biological media and form aggregates/agglomerates or protein corona which could give non-representative toxicity results.

\subsection{Nanotoxicology of $\mathrm{CeO}_{2}$}

Cerium oxide nanoparticles are widely used as catalysts in automotive industry, components in the fuel cells, or hydrogen production in energy-related industry, additives for consumer products like sunscreen, drug carriers in the pharmaceutical industry, and as abrasive particles for semiconductor industry [44, 45]. The mass density of $\mathrm{CeO}_{2}$ is $7.215 \mathrm{~g} / \mathrm{cm}^{3}$ [24]. The variety of applications for nano-cerium oxide warrants occupational health studies to ensure safety of production, use, and disposal throughout the life cycle. Literature studies have shown that the main route of exposure for cerium oxide NPs is via inhalation [28-30, 44-46].

Nano-cerium oxide exerts both protective and toxic effects due to its oxidative properties [44, 45]. Cerium oxide NPs can scavenge radicals, which lowers the amount of ROS present in cells. 
However, cerium oxide can also increase cytotoxicity by increasing the intracellular hydrogen peroxide levels as well as increasing the ROS levels in some studies [44]. Red-ox reactions involving $\mathrm{Ce}^{2+}$ ions were proposed by Korsvik et al. to explain the possible mechanism of ROS formation [47]:

$$
\begin{gathered}
\mathrm{O}_{2}^{\cdot-}+\mathrm{Ce}^{4+} \rightarrow \mathrm{O}_{2}+\mathrm{Ce}^{3+}, \mathrm{E}_{0}=1.61 \mathrm{~V} \\
\mathrm{O}_{2}{ }^{--}+\mathrm{Ce}^{3+}+2 \mathrm{H}^{+} \rightarrow \mathrm{H}_{2} \mathrm{O}_{2}+\mathrm{Ce}^{4+}, \mathrm{E}_{0}=-2.34 \mathrm{~V}
\end{gathered}
$$

Several studies have demonstrated contradicting results whether nano-cerium oxide is related to the synthesis method, particle size, and cell type [24, 44, 45]. It was shown that the temperature can influence the size and subsequently the zeta potential of the NPs [48]. This in turn affects the aggregation behavior of the NPs as well as their cytotoxicity. Another study showed that the difference in synthesis temperature can influence the size of the NPs which could in turn lead to a higher $\mathrm{Ce}^{3+} / \mathrm{Ce}^{4+}$ ratio on the surface of the particle as the size of the of the NP decreases.

As is the case for silica, the cytotoxicity of nano-cerium oxide can also involve the formation of ROS, which, in excess, can increase the cellular stress and decrease cell viability.

An in vivo study showed a time-dependent increase in oxidative stress in the spleen and liver as well as the presence of hepatic granuloma when male Sprague-Dawley rats were infused intravenously with $70 \mathrm{mg} / \mathrm{kg}$ (30 nm cerium NPs) [49]. Another study demonstrated that cerium oxide NPs were able to induce DNA damage in leukocytes and liver cells as well as form micronuclei in the bone marrow and blood cells when female Wistar rats ingested $1000 \mathrm{mg} / \mathrm{kg}$ of $25 \mathrm{~nm}$ cerium oxide NPs [50]. The study also established a dose-, time-, and organ-dependent relationship with the bioaccummulation of cerium oxide NPs after performing histopathological analysis.

In summary, cerium oxide NPs can exhibit both adverse and protective effects. This depends on the synthesis method, the cell types being studied, and possibly the particle size, although the latter should be studied in more detail as at present is not possible to draw a definitive conclusion. As is the case for silica, standardized tests should be performed so that the results will be more comparable and a more definitive conclusion can be made. Nanoceria is suspected to have CMR properties; however, this still needs to be demonstrated in vivo in relevant doses.

\subsection{Nanotoxicology of $\mathrm{Al}_{2} \mathrm{O}_{3}$}

Nano-aluminim oxide (alumina) has been used in various applications ranging from being components in cements, acting as catalysts and surface coatings, to being abrasive particles in semiconductor production [46, 51]. The mass density of $\mathrm{Al}_{2} \mathrm{O}_{3}$ is $3.987 \mathrm{~g} / \mathrm{cm}^{3}$ [24]. Despite their wide use, alumina NPs are the least studied of the three nanoparticles presented so far. This is reflected by the limited number of toxicological studies for alumina NPs as well as the absent of an OECD dossier for alumina NPs. As is the case for most NPs, the most common route of exposure for nano-alumina is via inhalation [28-30, 46, 51].

Several studies have shown that alumina NPs are also able to induce excessive ROS production which could in turn damage other cellular compartments like the plasma membrane and 
mitochondria, thus decreasing the cellular activity and/or viability. Moreover, excessive intracellular ROS could interact with the cellular genetic material that could further lead to mutations [6-8, 28, 30, 37, 52, 53]. According to present toxicological reports [51, 54-57], alumina NPs are less cytotoxic than NPs, such as cerium oxide, gold, silver, or copper oxide NPs, but they may be also capable of inducing sufficient amount of ROS to decrease cell viability. In addition, it was shown that nano-alumina can cause impairments to the cellular innate defense mechanisms against airborne pathogenic organisms [58]. Similarly, another in vitro study demonstrated that RAW264 macrophages that were incubated with $13 \mathrm{~nm}$ alumina NPs at 200 and $400 \mu \mathrm{g} / \mathrm{mL}$ for 72 hours exhibit changes in the morphology as well as a dose-dependent cytotoxicty which results in the decrease in cell viability by up to $40 \%$ of the control [54].

It should be noted that the choice of cell line used can also influence the outcome of the toxicological assay [51]. In this study, six cell lines (BEAS-2B representing the lung, Chang for the liver, HaCaT for the skin, H9C2 for the heart, T98G for the brain, and HEK-293 for the kidney) were exposed to 5 and $20 \mu \mathrm{g} / \mathrm{mL} 180-200 \mathrm{~nm}$ of nano-alumina for 48 hours. The results showed that there is a dose-dependent decrease in the viability for all six cell lines ranging from a $15 \%$ decrease to as much as $50 \%$. The most sensitive cell line was $\mathrm{HaCaT}$ and BEAS-2B.

In summary, nano-alumina has been shown to be able to induce cytoxic damages via the producion of ROS which in turn reduce the cell viability. However, the number of articles focusing on cytotoxicity of alumina NPs and the exact mechanism of toxicity is very limited and nonexistent, respectively. As in the case of mutagenicity, conflicting reports of alumina NPs render their possession of CMR properties inconclusive. The difference between toxicological studies can possibly be explained by the synthesis method or the cell culture conditions or differences in cell types sensitivity. Therefore, standardized tests should be conducted so that different results become more comparable.

\subsection{Nanotoxicology of CNTs}

Carbon nanotubes are believed to fit the fiber paradigm on par with asbestos [28-30, 46]. In summary, the fiber paradigm refers to any (nano)materials with:

- $\quad$ Diameter less than $3 \mu \mathrm{m}$

- Length of more than 10-20 $\mu \mathrm{m}$

- $\quad$ Aspect ratio greater than 3

- Aerodynamic diameter of less than $10 \mu \mathrm{m}$

- $\quad$ Biopersistent and rigid

Therefore, many toxicological studies have been conducted so far [28-30, 46, 59-63]. Published in vivo studies have shown that CNTs can enter the body via several pathways including inhalation, oral uptake, and dermal uptake. Many of these articles focus on the inhalation or intratracheal instillation; however, the most relevant exposure route to humans based on the electronic and biomedicine applications of CNTs would be oral uptake or intraveneous injection [60,61]. The studies based on the latter two exposure routes are, unfortunately, presently quite limited. 
The mechanism of CNTs toxicity is believed to be also linked to the excessive ROS production induced by oxidative stress [59-63]. Due to size limitations, this chapter will not discuss in vitro studies. Interested readers are directed to [61] for an overview.

An in vivo intratracheal instillation study showed that mice that were instilled with 0,1 , and $0.5 \mathrm{mg}$ of SWCNT exhibited granulomas after 7 days with a dose-dependent relation and severe inflammation in the lungs after 90 days [59]. The dimensions of the SWCNT are reported as aerodynamic diameters. A 9-day intratracheal instillation study on F344 rats was conducted where $0.5 \mu \mathrm{g} / \mathrm{ml}$ MWCNTs was given five times a day [63]. The results showed hyperplastic lesions and inflammations in a dose-dependent manner. The MWCNT was of $13 \mu \mathrm{m}$ in length and more than $50 \mathrm{~nm}$ in diameter. Interestingly, an inhalation study where C57BL/6 mice were exposed to maximally $5 \mathrm{mg} / \mathrm{m}^{3}$ MWCNTs for 14 days showed no inflammation in the lung or any other tissue damage [64]. Finally, the study conducted by Poland et al. [62] demonstrated that MWCNTs that were injected intraperitoneally at a dosage of $100 \mu \mathrm{g} / \mathrm{ml}$ into the body of the C57Bl/6 mice for up to 7 days induced asbestos-like effects such as inflammation and granulomas. The MWCNT samples used in the study were quite polydisperse, having a diameter ranging from 10 to $165 \mathrm{~nm}$ and length ranging from 1 to $56 \mu \mathrm{m}$.

In summary, most in vivo toxicological studies for CNTs concur on the fact that the mechanism of CNT toxicity is linked to overproduction of ROS. This property can be exacerbated or mitigated by the specific physicochemical properties of the CNTs [60, 61]. Although many articles exist on the toxicological studies of CNTs, the results obtained are difficult to be compared to each other due to the lack of standard characterization of CNTs as well as the protocol for exposure and cytotoxic assays. As for the CMR properties of CNTs, different studies suggested that CNTs may exhibit genotoxic properties and, hence, more standardized studies should be conducted to yield more comparable results between different studies so that a definite conclusion can be made.

\subsection{Nanotoxicology of graphene}

Graphene-based nanomaterials are the most novel group of nanomaterials covered in this review. In comparison to CNTs, toxicological studies of graphene-based nanomaterials are less but gradually increasing and are less coherent in terms of their conclusions [65-68]. This can be most likely attributed to differences in synthesis methods. As is the case with the metallic nanoparticles and the CNTs, the most probable routes of exposure are inhalation, followed by oral and dermal routes [28-30, 46, 65, 66]. It should be noted that the available in vivo studies up to date have focused on intravenous and oral administration with the intent of studying the mechanistic effects and biodistribution rather than relating to the relevancy of occupational exposures.

Analogous to the in vitro studies, most in vivo studies also showed a dose-dependent toxicity for the exposure of graphene-based nanomaterials. Zhang et al. conducted an intravenous injection of GO, with the size of 100-800 nm and a thickness of $1 \mathrm{~nm}$, into Kun Ming mice at a dose of 1 and $10 \mathrm{mg} / \mathrm{kg}$ [69]. After 14 days, inflammation and the formation of edemas and granulomas were observed during histopathological analysis in a dose-dependent manner. The studies of Singh et al. demonstrated how functionalization can influence the degree of 
toxicity of the graphene oxides [70, 71]. Pristine GO and GO functionalized with amine (NH2) having size range between 0.2 and $5 \mu \mathrm{m}$ were injected intravenously into Swiss male mice at a concentration of $250 \mu \mathrm{g} / \mathrm{kg}$. Histopathological analysis was conducted after $15 \mathrm{~min}$ and found that mice injected with aminated GO showed no sign of thrombotoxicity, whereas on the other hand, mice injected with GO showed thrombotoxicity as well as aggregation of blood platelets in as much as $48 \%$ of the lung blood vessels. Finally, an oral uptake study in ICR mice showed atrophic characteristics in all major organs as well as lowered weight of the body and tail length [36]. The mice were fed with 0.05 and $0.5 \mathrm{mg} / \mathrm{ml}$ of GO (size of $2000 \mathrm{~nm}$ and height of $1.8 \mathrm{~nm}$ ) in drinking water.

Studies on the CMR properties of graphene-based materials are very limited. Bengtson et al. conducted an intratracheal instillation study with GO and multilayer reduced GO into C57BL/ $6 \mathrm{~J}$ mice at a dose of $18 \mathrm{up}$ to $162 \mu \mathrm{g} /$ mouse. Analysis was performed at various time points during the 90-day study period [72]. In addition to inflammatory responses, it has been observed that both GO and reduced GO were able to cause DNA damage in the BAL cells at the lowest dose of $18 \mu \mathrm{g} /$ mouse from day 3 of the study. However, DNA damage was not significantly observed in the lungs or liver. Another study demonstrated micronuclei formation in B and T lymphocytes as well as the primary lymphocytes when these cell lines were exposed to $6.25-400 \mu \mathrm{g} / \mathrm{ml}$ of GO (size ranging from one to tens of $\mu \mathrm{m}$ ) [73].

The toxicological mechanisms of graphene-based nanomaterials are believed to be related to the excessive formation of ROS [65-68]. When internalized within a cell, graphenes could disrupt the electron transport chain which causes the production of excess peroxide and hydroxyl radicals. Subsequently, the homeostasis of the intracellular reactive oxidative species is disrupted. Additionally, graphenes may cause membrane disruptions as well as damage the cell integrity directly by their sharp edges $[65,66]$. Nevertheless, the exact mechanism of graphene-based nanotoxicoloy is still largely unknown [65-68].

In vivo studies suggest that graphenes could have been taken up and retained in various organs for a prolonged period of time. However, most in vivo studies focused on explaining the mechanism of the toxicity and did not take the realistic dosage or route of exposure into account [66]. Hence, more uniform in vivo studies with regard to occupational settings will have to be conducted in the future in order to draw a definite conclusion from these studies. Finally, limited number of genotoxic studies suggested that graphenes are capable of interacting with DNA and could hold genotoxic properties.

\subsection{Overview of the nanotoxicological properties of studied ENM}

There is an emerging consensus in literature that the toxicity of engineered nanomaterials seems to be higher than the toxicity of bulk material (review in [74]). The toxicodynamic mechanisms are summarized in Table 3. Furthermore, an insufficient hazard characterization leads to limited availability of data on physicochemical properties, (eco)toxicological properties, and the environmental fate information, all of which are prerequisites for comprehensive and quantitative risk assessment [52]. According to Pietroiusti et al. [74], the following knowledge gaps of ENM can be listed at present: 


\begin{tabular}{lll}
\hline ENM & OEL $\left[\mathbf{1} / \mathrm{cm}^{3}\right]$ & Source \\
\hline $\mathrm{SiO}_{2}$ & 40,000 & IFA, SER \\
$\mathrm{CeO}_{2}$ & 20,000 & IFA, SER \\
$\mathrm{CNT}$ & 0.01 & IFA, SER, BSI \\
\hline
\end{tabular}

IFA, German Social Accident Insurance (Germany); SER, Social and Economic Council (Netherlands); BSI, British Standards Institute (UK).

Table 3. Summary of the toxicological mechanisms of target ENM and proposed OEL.

- Data gaps in toxicokinetics of ENM in organisms and cells

- Insufficient understanding of the mechanisms of toxicity

- Nano-specific biomarkers of ENM toxicity or ENM-induced diseases

- $\quad$ Predictive models of ENM toxicity

All this leads to impossibility of establishing OEL for the predominant majority of ENMs rendering quantitative risk assessment for nanomaterials impossible.

The OELs for bulk parent materials can significantly differ from their nano-counterparts due to the discussed differences in their physicochemical properties. The complexity also arises from the fact that the best metric (by mass, concentration, or number) for nanoparticle exposure characterization is still debatable [75-77]. OELs for nanomaterials are available only in few cases (see Table 3).

Due to this situation, the concept of OEL has been replaced by nano-reference value (NRV), however, without regulatory significance. Such NRVs can be sector or organization specific. Furthermore, quality of available data can be also problematic. This is a concern already recognized in literature [76, 78]. These recent publications have demanded for a uniform standard operating procedure so that results obtained from different studies can be compared faithfully. Such standardized protocols should meet the criteria of the nanomaterial testing program coordinated by OECD [41].

\begin{tabular}{lll}
\hline Target & Mechanism & Type of NP \\
\hline Plasma membrane & Mechanical action, ROS & $\mathrm{SiO}_{2}, \mathrm{CeO}_{2}$, graphenes \\
Lysosomes & Receptor activation & $\mathrm{SiO}_{2}, \mathrm{CeO}_{2}, \mathrm{CNT}^{-}$ \\
Mitochondria & Membrane disruption & $\mathrm{CeO}_{2}$ \\
Nucleus & Membrane disruption, protein downregulation & $\mathrm{SiO}_{2}$ \\
& Transcription inhibition & $\mathrm{SiO}_{2}$ \\
Cytosol proteins & Inhibition of cytokines & $\mathrm{CNT}^{2}$ \\
& Actin & $\mathrm{SiO}_{2}$ \\
& CYP450 & $\mathrm{SiO}_{2}$ \\
\hline
\end{tabular}

Contents are based on Refs. [74, 79]. 


\section{Elements of nanomaterial risk assessment}

The process of chemical or nanomaterial risk assessment is conducted in order to estimate the risks associated with a particular operation and materials. In a further step, the risk assessment derives a set of protective measures that allow for reduction of the risk for the workers and the environment. The availability of the toxicological data defines which type of risk assessment method can be used. If extensive data is available, quantitative risk assessment can be used to derive OELs for a particular material. Subsequently, the emission could be controlled to confirm that the exposure does not exceed the predetermined OELs. Almost by definition, this is not the case for novel ENM, which may bring about unanticipated interactions that alter their safety profile compared to the bulk material.

Challenges in the traditional chemical risk assessment approach can be traced to the assumption that the hazard and the risk can be quantified in an absolute way [86]. In contrast, since hazard profile data for novel material are inherently uncertain, the risk can be estimated only in a relative way. Such reasoning is supported also by the facts that there are conventional strong anthropogenic sources of nanomaterials, such as wax candles, radiator, frying, burning cigarettes, and traffic [87]. A way to estimate the risk is by grouping of materials based on certain similarity metrics. Such an approach favors categorical risk assessment tools, which result in a classification into a hazard and control band for the process under investigation (Table 4).

Control banding was developed in the pharmaceutical industry as a pragmatic tool to manage the risk resulting from exposure to a wide variety of potentially hazardous substances in the absence of firm toxicological and exposure data [88]. Its applications to safety of nanomaterials have been reviewed in [89]. The control banding approach is based on two pillars: the fact that there are a limited number of control approaches and that many problems have been met and

\begin{tabular}{lllll}
\hline Tool & Hazard bands & Exposure bands & Risk bands & References \\
\hline ANSES & 5 & 4 & 5 & {$[80]$} \\
CB Nanotool & 4 & 4 & 4 & {$[81]$} \\
ISO & 5 & 4 & 5 & {$[82]$} \\
Imec & 3 & 4 & 3 & {$[83]$} \\
Nano & & & & {$[84]$} \\
Stoffenmanager & 5 & 4 & 3 & {$[85]$} \\
NanoSafer & 4 & 5 & 5 & \\
\hline
\end{tabular}

* Scores from 0 to 100 .

*** Score ranges.

ISO, ISO Technical Standard ISO/TS 12901-2:2014 “Nanotechnologies-Occupational risk management applied to engineered nanomaterials".

Table 4. Summary of control banding tools. 
solved before. The second pillar assumes that risks are at least qualitatively similar, even if no numerical probabilities can be assigned to them.

\subsection{Hazard banding}

For most cases, control banding tools follow a decision tree approach to characterize the hazards. The ISO Technical Standard 12901-2:2014 "Nanotechnologies-Occupational risk management applied to engineered nanomaterials" (in short ISO tool) uses only few physicochemical parameters of the NPs, namely, the water solubility and fibrocity, as preliminary questions to decide whether the NPs can be classified. Eventually, the NPs will be designated into one of the five classes of hazard bands $(\mathrm{HB})$ based on the available toxicological data. In the case where the toxicological data are insufficient or unknown, the ISO tool makes use of the hazard band of the bulk or an analogous substance with an additional penalty. Similarly, the hazard banding for ANSES also follows a decision tree. Three preliminary questions are used to identify the "nanorelevance" and to determine whether the use of the ANSES tool is warranted. Interestingly, according to the ANSES tool, a persistent fiber will automatically be designated the highest $\mathrm{HB}$ of 5 . This is also the case for the ISO decision tree. Subsequently, a substance of reference (be it a bulk or an analogous material) is used in order to assign a hazard band with additional penalties depending on the physicochemical properties like water solubility and chemical reactivity in the nanoform. One point of interest is that the ANSES tool does not include toxicological information, such as acute toxicity or the CMR properties when designating hazard bands.

The hazard banding of Stoffenmanager Nano tool follows a tiered approach which results in a decision tree. If the material is "nano-relevant," the decision tree can be followed to the next tier. Subsequently, physicochemical properties of the nanomaterials including the water solubility, fibrocity, as well as the toxicological information are used to designate a hazard band for the material of interest. In the case where the toxicological information of the nanomaterial is not known, Stoffenmanager Nano refers to the data available for the ENM studies conducted by OECD, or if the material of interest is not ranked or included within the OECD framework, the toxicological information from the bulk materials will be used.

The hazard banding for the Imec tool is a questionnaire assessment. Several physicochemical parameters are collected, such as solubility, persistency, and dispersibility in water, the size, and morphology and the toxicological information of the nanomaterial. The CB Nanotool sums points based on severity factors which are related to both the physicochemical properties and the toxicological information in the nanoform as well as the bulk form. Of the maximum 100 points, a maximum of 70 points are designated for nanoform properties, while the other remaining 30 points are for the bulk material properties. Every set of severity factors contains a questionnaire-type questions which, depending on the answer, will result in points that will be accumulated for the hazard banding. When a property is unknown, $75 \%$ of the maximum points for that property will be allocated.

LICARA Nanoscan has a different characterization approach. It provides risk benefit/analysis but not a definite risk categorization. The risks score is shown on a scale with $0-0.33$ being low 
risk, $0.33-0.67$ being medium risk, and $0.67-1$ being high risk. LICARA Nanoscan assumes the worst-case scenario when a question is not answered or the detail is unknown.

Finally, the NanoSafer approach for hazard assessment first takes into account whether the nanomaterial has a high aspect ratio, in which case the highest hazard is attributed. Other factors, such as the surface modification and the OEL for the analogous material, also contribute to the designation of a hazard factor that will be later used in the calculation of the hazard score. Finally, additional input physicochemical information asked by NanoSafer includes the dimensions, the specific density, the specific surface area, powder dustiness, and, most importantly, the hazard sentences. The hazard assessment of NanoSafer incorporates accumulative sum of the hazard score from the hazard sentences found on the safety data sheet of the nanomaterial.

\subsection{Exposure banding}

The exposure characterization for the ISO tool also follows a tiered approach which results in a decision tree. However, unlike hazard banding, there are several decision trees available, and one must select the most relevant one depending on the physical state of the NPs (embedded in a solid matrix, dispersed in liquid suspension or as nanopowders) or based on the manufacturing process. The end points will be given in exposure bands 1-4, where 4 represents the highest exposure probability. Similarly, the banding of the emission potential for ANSES tool depends largely on the physical state of the nanosubstance where aerosols would result in the highest emission potential.

The exposure banding for the Stoffenmanager Nano comprises different factors, each with its own reference table. The factors taken into account for the exposure include information about the substance, handling, location of the emission (near field or far field), local controls for the emission source, dispersion/transmission conditions, receptors (present of personal enclosure and/or protection equipment), background exposure, duration, and frequency. Stoffenmanager Nano requires, by far, the most extensive input parameters in order to determine the exposure band, specifically 26 inputs in total [90].

For the exposure estimation of the Imec tool takes several factors into account, including the localization (far or near field) and duration of the manipulation involving the ENM and the amount used. The exposure banding for the CB Nanotool assigns scores based on different probability factors related to the manipulation and scenario which involves the nanomaterial of interest. A maximum probability score of 100 can be summed based on the answers given to the questions pertaining to the probability factors. An unknown answer will result in a $75 \%$ allocation of the maximum point for that probability factor.

The exposure characterization for NanoSafer is calculated using the emission rate, the default activity energy factor, and the mass flow/amount used in the process. Both the convection and the rate of ventilation are taken into account for the near-field and far-field model calculation [85]. 


\subsection{Control banding}

The output of a control banding tool is a recommended set of measures which reduce the potential of exposure. For example, the ISO standard provides the following groups:

Control Level 1. Natural or mechanical ventilation.

Control Level 2. Local ventilation-extractor hood, table hood, etc.

Control Level 3. Enclosed ventilation - fume hood, biosafety cabinet, ventilated booth, etc.

Control Level 4. Full containment (continuously closed systems).

Control Level 5. Full containment and review by a safety specialist.

Some tools, for example, LICARA Nanoscan, only provide a decision support scheme (whether to continue with this nano-product or not) or, such as Stoffenmanager Nano, only give prioritization on the task of concern.

\subsection{Comparison of the input parameters of used control banding tools}

The hazard parameters of so-described tools are summarized in Table 5. All control banding tools required the primary particle diameter of the NP, solubility in water, and the fibrocity/ aspect ratio. Most tools demand the CMR and toxicity information but differ in whether this information should be taken from the the nano- or the bulk form. This highlights the fact that risk assessment is a very dynamic process that can always change depending on the availability of data and that different control banding tools should be compared for the process of interest to have a well-rounded risk assessment.

Among all control banding tools presented here, the approach of Imec took into account the most physicochemical properties in order to assign hazard bands with a total of nine physicochemical parameters as inputs. On the other hand, the ISO standard recommends collection of much more parameters for future use. In view of the information presented so far, this can be considered as a shortcoming since most of the prescribed parameters are not readily available in the material specifications and safety data sheets.

For the case of toxicological information, no single property is shared by all control banding approach. The CB Nanotool required most toxicological information with a total of up to 12 input parameters, while the ANSES control banding approach required just one. Interestingly, not all CB tools agree on the question of whether toxicological information is needed from the nanoform or from the bulk form. The ISO standard and the CB Nanotool required both the nanoform and the bulk material toxicological information in order to assign a hazard band. This is unrealistic for the state of the art at present.

The Stoffenmanager Nano and LICARA Nanoscan only require the nanoform toxicological information, while the Imec approach, NanoSafer, and the ANSES CB tool only require toxicological profile of the parent form. According to the 2016 evaluation report of OECD, the CMR properties as well as the acute, subacute, chronic, or specific organ toxicity are rarely known 


\begin{tabular}{|c|c|c|c|c|c|c|c|}
\hline Parameter & ISO & ANSES & NSM$^{* *}$ & Imec & Nanotool & LICARA & NanoSafer \\
\hline & \multicolumn{7}{|c|}{ Physicochemical parameters ${ }^{*}$} \\
\hline Reactivity/surface chemistry & & Y & & Y (bulk) & Y & Y & Y \\
\hline Aspect ratio/fibrocity & $\mathrm{Y}$ & Y & $\mathrm{Y}$ & $\mathrm{Y}$ & $\mathrm{Y}$ & $\mathrm{Y}$ & Y \\
\hline Particle diameter & $\mathrm{Y}$ & Y & Y & $\mathrm{Y}$ & $\mathrm{Y}$ & $\mathrm{Y}$ & Y \\
\hline Solubility & $\mathrm{Y}$ & Y & $\mathrm{Y}$ & Y (bulk) & $\mathrm{Y}$ & Y & Y \\
\hline Biopersistency & & Y & & Y (bulk) & & & \\
\hline Dispersion capacity & & & & Y (bulk) & & & \\
\hline Aggregation/agglomeration & & & & $\mathrm{Y}$ & & & \\
\hline \multirow[t]{2}{*}{ Physical form } & & & & $\mathrm{Y}$ & & & \\
\hline & \multicolumn{7}{|c|}{ Toxicological parameters* } \\
\hline Dermal & & & $\mathrm{Y}$ & & Y & Y & Y (bulk) \\
\hline Acute & $\mathrm{Y}$ & & $\mathrm{Y}$ & & Y & $\mathrm{Y}$ & \\
\hline Acute, bulk & Y & Y & & $\mathrm{Y}$ & Y & & $\mathrm{Y}$ \\
\hline CMR, bulk & & & & Y & Y & & Y \\
\hline CMR & $\mathrm{Y}$ & & Y & & Y & Y & \\
\hline
\end{tabular}

${ }^{*}$ Concerning nanoform if not stated otherwise.

**NSM, Stoffenmanager Nano; ISO, ISO Technical Standard ISO/TS 12901-2:2014 “Nanotechnologies-Occupational risk management applied to engineered nanomaterials"; CMR, carcinogenic, mutagentic, and reprotoxic.

The number of hazard parameters required to completed the ISO tool is less than those of the approach of Imec and the CB Nanotool but still more than those of ANSES and Stoffenmanager Nano. The total number of hazard parameters required is the same for the ISO tool as for NanoSafer and LICARA Nanoscan.

Table 5. Summary table of input parameters.

for the nanoforms [41]. Therefore, this implies that users will be more likely to take the unknown penalty while completing the control banding approaches that require nanoform toxicological information which can differ in the degree of conservativeness from tool to tool. For instance, the CB Nanotool assigns $75 \%$ of the severity score for unknown information, while LICARA Nanoscan assumes the worst-case scenario in the case where a question is left unanswered.

In conclusion, different CB tools emphasize differently on the parameters taken into account for the hazard characterization. The CB Nanotool requires extensive toxicological information of the nano- and the bulk form, while the Imec approach focuses more on the physicochemical properties of the nanomaterial for the hazard banding assessment.

\subsection{The NanoStreeM's tiered risk assessment framework}

The NanoStreeM consortium derived a list of activities that can potentially cause the release of nanoparticles [91]. Described activities include, among others, processes from the operation and cleaning and maintenance of certain processing tools. Based on this survey, the consortium further developed a guidance for performing nano-risk assessment for the semiconductor 


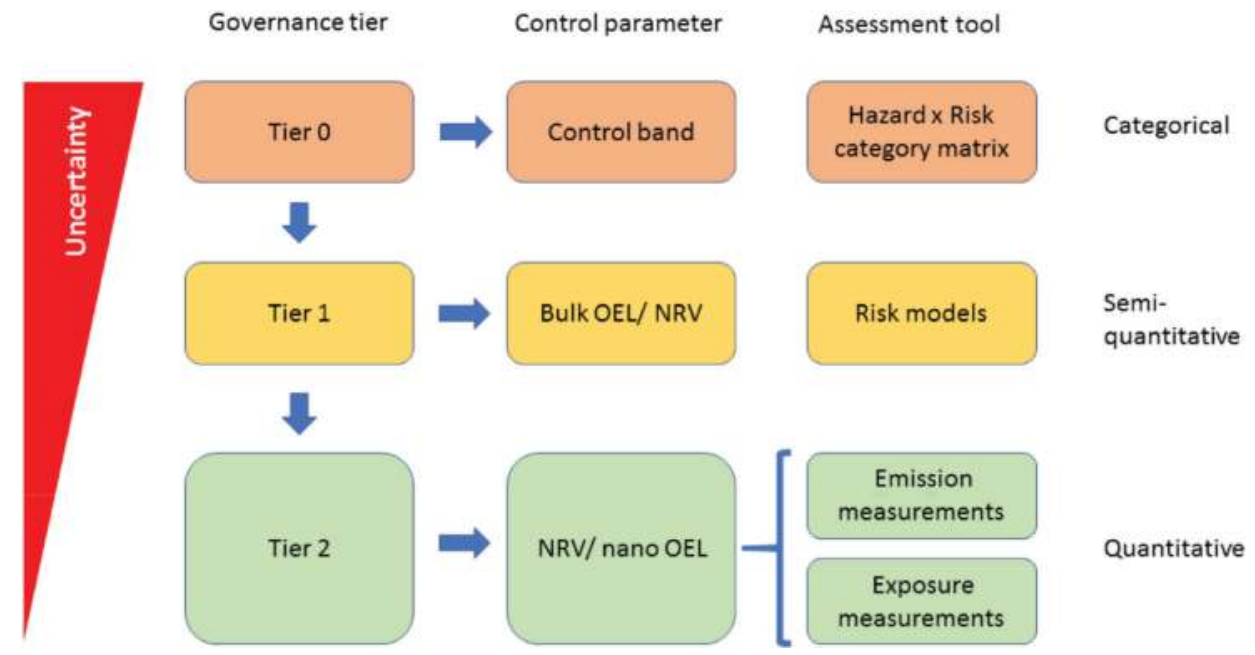

Figure 1. Generalized tiered risk assessment approach.

industry (Deliverables 3.1, [4]). The framework proposed a tiered approach, based on the principles of prior OECD work, to identify specific scenarios or tasks that warrant further detailed risk assessment. According to the guidance prior to the start of the risk assessment, characterization information of the substance, the toxicological information, and the use scenario should be well described. On the other hand, performing complete physicochemical characterization and toxicological studies of the nanomaterial is not always feasible or desirable since the results are not likely to be applicable in other contexts or settings as well as being costly and time-consuming. Therefore, there is a need for flexible frameworks that can give the user a focusing point onto which task or use scenario most urgently requires further hazard and exposure characterization.

The initial tiered approach framework can be further generalized (Figure 1) as to include future versions of so-described risk assessment tools or even novel sector-specific tools. As the amount and quality of information improve, the risk assessment can gradually proceed from control bands to NRV and finally regulatory relevant nano-OELs. This can be achieved following the precautionary principle without compromising the necessary safety measures even if the hazard characterization information is uncertain.

The three tiered risk assessment methodologies can be described as follows:

Tier 0 gives an overall screening of the situation including hazard and exposure assessment. The output is a categorical ranking of prioritization for the subsequent Tiers 1 and 2. As Tier 0 method, the consortium has identified the ISO Technical Standard.

Tier 1 gives a semiquantitative result for the exposure and the hazard assessment to further elucidate the hotspots. Tier 1 consists of application of a risk model, providing refinement of the Tier 0 result in the case of an identified concern. The outcome of this tier is an indicative 
estimate of the expected exposure (i.e., a NRV) and a prescription on how the exposure can be controlled.

Tier 3 requires the use of actual exposure measurement or toxicological data for risk assessment. Tier 2 consists of designating of specific monitoring and control strategies for the exposure or emission of nanomaterials, which validates or refutes the estimate of Tier 1 and also refines the identified control strategies.

\section{Conclusions}

This chapter provides an overview of several control banding tools for risk assessment of ENM. Their application to semiconductor production processes has been presented as a preliminary use case in view of the information collected in the NanoStreeM project. While the page count limitations do not allow for thorough overview of nanoparticle toxicology, identified gaps in the state of the art demonstrate the main advantages and limitations of the different control banding tools. Substantial knowledge gaps can be identified for even widely used by the industry ENM, such as $\mathrm{CeO}_{2}$ and $\mathrm{Al}_{2} \mathrm{O}_{3}$ nanoparticles. The situation is even worse for materials with promising nanoelectronic applications, such as CNTs and graphenes. Furthermore, it was found that the ISO Technical Standard ISO/TS 12901-2:2014 needs further clarification in order to improve its usability. The presented NanoStreeM generalized tiered risk assessment approach allows for the use of different, possibly even sector-specific tools, in combination with emission or exposure measurement field studies.

\section{Acknowledgements}

Authors declare no conflict of interest. The work is supported by the NanoStreeM project, funded under H2020 grant agreement 688194 of the European Commission. The authors would like to acknowledge the helpful remarks of Dr. Lieve Geerts and Dr. Maaike Le Feber.

\section{List of acronyms}

CB control canding

CNT carbon nanotube

CMOS complementary metal-oxide-semiconductor

CMP chemical mechanical planarization, chemical mechanical polish

SWCNTs single-walled carbon nanotubes

DWCNTs double-walled carbon nanotubes

MWCNTs multiwalled carbon nanotubes 
ENM engineered nanomaterials

NP nanoparticle

GO graphene oxide

CMR carcinogenic, mutagentic, and reprotoxic

QD quantum dots

ROS reactive oxidative species, reactive oxygen species

OEL occupational exposure limit

OECD Organization for Economic Co-operation and Development

NRV nano-reference value

\section{Author details}

Ponnapat Watjanatepin and Dimiter Prodanov*

*Address all correspondence to: dimiterpp@gmail.com

EHS, Imec, Belgium

\section{References}

[1] Schulte PA, Geraci CL, Hodson LL, Zumwalde RD, Kuempel ED, Murashov V, Martinez $\mathrm{KF}$, Heidel DS. Overview of risk management for engineered nanomaterials. Journal of Physics: Conference Series. 2013;429(1):012062

[2] Bystrzejewska-Piotrowska G, Golimowski J, Urban P. Nanoparticles: Their potential toxicity, waste and environmental management. Waste management (New York N.Y.). 2009; 29:2587-2595

[3] Vargas Ferreira F, De Simone Cividanes L, Sales Brito F, Rossi Canuto de Menezes B, Franceschi W, Nunes Simonetti E, Patrocnio Thim G. Functionalizing Graphene and Carbon Nanotubes. Switzerland AG: Springer International Publishing; 2016

[4] Lehman S, Morris A, Mueller P, Salem A, Grassian V, Larsen S. Silica nanoparticlegenerated ROS as a predictor of cellular toxicity: Mechanistic insights and safety by design. Environmental Science: Nano. 2016;3(1):56-66

[5] Speed D, Westerhoff P, Sierra-Alvarez R, Draper R, Pantano P, Aravamudhan S, Loon Chen K, Hristovski K, Herckes P, Bi X, Yang Y, Zeng C, Otero-Gonzalez L, Mikoryak C, Wilson B, Kosaraju K, Tarannum M, Crawford S, Yi P, Liu X, Babu SV, Moinpour M, Ranville J, Montano M, Corredor C, Posner J, Shadman F. Physical, chemical, and in vitro toxicological characterization of nanoparticles in chemical mechanical planarization 
suspensions used in the semiconductor industry: Towards environmental health and safety assessments. Environmental Science: Nano. 2015;2(3):227-244

[6] Buzea C, Pacheco I, Robbie K. Nanomaterials and nanoparticles: Sources and toxicity. Biointerphases. 2007;2(4):MR17-MR71

[7] Oberdörster G. Safety assessment for nanotechnology and nanomedicine: Concepts of nanotoxicology. Journal of Internal Medicine. 2010;267(1):89-105

[8] Luyts K, Napierska D, Nemery B, Hoet PHM. How physico-chemical characteristics of nanoparticles cause their toxicity: Complex and unresolved interrelations. Environmental Science: Processes \& Impacts. 2013;15(1):23-38

[9] Shepard MN, Brenner S. An occupational exposure assessment for engineered nanoparticles used in semiconductor fabrication. The Annals of Occupational Hygiene. 2013;58(2):251-265

[10] Forsberg M, Olsson J. Chemical mechanical polishing for surface smoothing. Physica Scripta. 2002;T101:200-202

[11] Zantye P, Kumar A, Sikder A. Chemical mechanical planarization for microelectronics applications. Materials Science and Engineering: R: Reports. 2004;45(3-6):89-220

[12] Singh R, Lee SM, Choi K-S, Basim G, Choi W, Chen Z, Moudgil B. Fundamentals of slurry design for CMP of metal and dielectric materials. MRS Bulletin. 2002;27(10):752-760

[13] Dekkers S, Oomen A, Bleeker E, Vandebriel R, Micheletti C, Cabellos J, Janer G, Fuentes N, Vázquez-Campos S, Borges T, João Silva M, Prina-Mello A, Movia D, Nesslany F, Ribeiro A, Leite PE, Groenewold M, Cassee F, Sips A, Dijkzeul A, van Teunenbroek T, Wijnhoven SWP. Towards a nanospecific approach for risk assessment. Regulatory Toxicology and Pharmacology. 2016;80:46-59

[14] Schnorr J, Swager T. Emerging applications of carbon nanotubes. Chemistry of Materials. 2011;23(3):646-657

[15] Zhao J, Buldum A, Han J, Ping Lu J. First-principles study of li-intercalated carbon nanotube ropes. Physical Review Letters. 2000;85(8):1706-1709

[16] Nguyen KT, Zhao Y. Integrated graphene/nanoparticle hybrids for biological and electronic applications. Nanoscale. 2014;6(12):6245-6266

[17] Peng L-M, Zhang Z, Wang S. Carbon nanotube electronics: Recent advances. Materials Today. 2014;17(9):433-442

[18] Chiang IW, Brinson BE, Huang AY, Willis PA, Bronikowski MJ, Margrave JL, Smalley RE, Hauge RH. Purification and characterization of single-wall carbon nanotubes (SWNTs) obtained from the gas-phase decomposition of CO (HiPco process). The Journal of Physical Chemistry B. 2001;105(35):8297-8301

[19] Liu Y, Zhao Y, Sun B, Chen C. Understanding the toxicity of carbon nanotubes. Accounts of Chemical Research. 2012;46(3):702-713 
[20] Ng S-H, Wang J, Wexler D, Konstantinov K, Guo Z-P, Liu H-K. Highly reversible lithium storage in spheroidal carbon-coated silicon nanocomposites as anodes for lithium-ion batteries. Angewandte Chemie International Edition. 2006;45(41):6896-6899

[21] Yin P, Kim T-H, Choi J-W, Lee K-B. Prospects for graphene-nanoparticle-based hybrid sensors. Physical Chemistry Chemical Physics. 2013;15(31):12785

[22] Brownson D, Kampouris D, Banks C. An overview of graphene in energy production and storage applications. Journal of Power Sources. 2011;196(11):4873-4885

[23] Gopal Sahoo N, Pan Y, Li L, Hwa Chan S. Graphene-based materials for energy conversion. Advanced Materials. 2012;24(30):4203-4210

[24] Hirst SM, Karakoti AS, Tyler RD, Sriranganathan N, Seal S, Reilly CM. Anti-inflammatory properties of cerium oxide nanoparticles. Small. 2009;5(24):2848-2856

[25] Murugadoss S, Lison D, Godderis L, Van Den Brule S, Mast J, Brassinne F, Sebaihi N, Hoet PH. Toxicology of silica nanoparticles: An update. Archives of Toxicology. 2017;91:2967-3010

[26] Napierska D, Thomassen L, Lison D, Martens J, Hoet P. The nanosilica hazard: Another variable entity. Particle and Fibre Toxicology. 2010;7(1):39

[27] Rasmussen K, González M, Kearns P, Riego Sintes J, Rossi F, Sayre P. Review of achievements of the OECD working party on manufactured nanomaterials testing and assessment programme. From exploratory testing to test guidelines. Regulatory Toxicology and Pharmacology. 2016;74:147-160

[28] ISO. Nanotechnologies - Health and Safety Practices in Occupational Settings Relevant to Nanotechnologies. iso/tr12885. Technical report, ISO; 2008

[29] Kosk-Bienko J, editor. Workplace Exposure to Nanoparticles. Luxembourg: EU-OSHA; 2009

[30] NIOSH, editor. Approaches to Safe Nanotechnology. USA: DHHS (NIOSH) Publication; 2006

[31] Abdal Dayem A, Hossain M, Lee S, Kim K, Saha S, Yang G-M, Choi H, Cho S-G. The role of reactive oxygen species (ROS) in the biological activities of metallic nanoparticles. International Journal of Molecular Sciences. 2017;18(12):120

[32] Fu C, Liu T, Li L, Liu H, Liang Q, Meng X. Effects of graphene oxide on the development of offspring mice in lactation period. Biomaterials. 2015;40:23-31

[33] Fu P, Xia Q, Hwang H-M, Ray P, Yu H. Mechanisms of nanotoxicity: Generation of reactive oxygen species. Journal of Food and Drug Analysis. 2014;22(1):64-75

[34] Kim K-A, Kim Y-H, Seok Seo M, Kyu Lee W, Won Kim S, Kim H, Lee K-H, Shin I-C, Han JS, Joong Kim H, Lim Y. Mechanism of silica-induced ROS generation in rat2 fibroblast cells. Toxicology Letters. 2002;135(3):185-191

[35] Le Feber M, Prodanov D, Zimmermann E. Identification of the most appropriate risk assessment methodologies for use in the semiconductor industry; 2017 
[36] Fubini B, Hubbard A. Reactive oxygen species (ROS) and reactive nitrogen species (RNS) generation by silica in inflammation and fibrosis. Free Radical Biology and Medicine. 2003;34(12):1507-1516

[37] $\mathrm{Hu}$ X, Cook S, Wang P, Hwang H-M. In vitro evaluation of cytotoxicity of engineered metal oxide nanoparticles. Science of the Total Environment. 2009;407(8):3070-3072

[38] Akhtar M, Ahamed M, Kumar S, Siddiqui H, Patil G, Ashquin M, Ahmad I. Nanotoxicity of pure silica mediated through oxidant generation rather than glutathione depletion in human lung epithelial cells. Toxicology. 2010;276(2):95-102

[39] Eom H-J, Choi J. Oxidative stress of silica nanoparticles in human bronchial epithelial cell, beas-2b. Toxicology in Vitro. 2009;23(7):1326-1332

[40] Hashimoto M, Imazato S. Cytotoxic and genotoxic characterization of aluminum and silicon oxide nanoparticles in macrophages. Dental Materials. 2015;31(5):556-564

[41] OECD. Physical-Chemical Properties of Nanomaterials Evaluation of Methods Applied in the OECD-WPMN Testing Programme. France: OECD Paris; 2016

[42] Kumari M, Indu Kumari S, Kamal S, Grover P. Genotoxicity assessment of cerium oxide nanoparticles in female wistar rats after acute oral exposure. Mutation Research/Genetic Toxicology and Environmental Mutagenesis. 2014;775-776:7-19

[43] van Broekhuizen P, van Veelen W, Streekstra W-H, Schulte P, Reijnders L. Exposure limits for nanoparticles: Report of an international workshop on nano reference values. The Annals of Occupational Hygiene. 2012;56:515-524

[44] Gagnon J, Fromm K. Toxicity and protective effects of cerium oxide nanoparticles (nanoceria) depending on their preparation method, particle size, cell type, and exposure route. European Journal of Inorganic Chemistry. 2015;2015(27):4510-4517

[45] Liguori B, Foss Hansen S, Baun A, Jensen KA. Control banding tools for occupational exposure assessment of nanomaterials - ready for use in a regulatory context? NanoImpact. 2016;2:1-17

[46] ANSES. Assessnment of the Risk Associated with Nanomaterials: Issues and Update of Current Knowledge. Maisons-Alfort, France: ANSES; 2014

[47] Korsvik C, Patil S, Seal S, Self WT. Superoxide dismutase mimetic properties exhibited by vacancy engineered ceria nanoparticles. Chemical Communications. 2007;10:1056

[48] Van Hoornick N, Prodanov D, Pardon A. Banding approach for engineered nanomaterial risk assessment and control. Journal of Physics: Conference Series. 2017;838:012017

[49] Yokel R, Unrine J, Wu P, Wang B, Grulke E. Nanoceria biodistribution and retention in the rat after its intravenous administration are not greatly influenced by dosing schedule, dose, or particle shape. Environmental Science: Nano. 2014;1(6):549-560

[50] Kumar A, Dhawan A. Genotoxic and carcinogenic potential of engineered nanoparticles: An update. Archives of Toxicology. 2013;87(11):1883-1900 
[51] Park E-J, Lee G-H, Yoon C, Jeong U, Kim Y, Cho M-H, Kim D-W. Biodistribution and toxicity of spherical aluminum oxide nanoparticles. Journal of Applied Toxicology. 2015; 36(3):424-433

[52] Brouwer D. Control banding approaches for nanomaterials. The Annals of Occupational Hygiene. 2012;56(5):506-514

[53] Kuhlbusch T, Asbach C, Fissan H, Göler D, Stintz M. Nanoparticle exposure at nanotechnology workplaces: A review. Particle and Fibre Toxicology. 2011;8(1):22

[54] Haynes WM, editor. CRC Handbook of Chemistry and Physics. 92nd ed. Boca Raton: CRC Press; 2011

[55] Yang H, Wu Q, Tang M, Kong L, Lu Z. Cell membrane injury induced by silica nanoparticles in mouse macrophage. Journal of Biomedical Nanotechnology. 2009;5:528-535

[56] Radziun E, Dudkiewicz Wilczyńska J, Książek I, Nowak K, Anuszewska EL, Kunicki A, Olszyna A, Ząbkowski T. Assessment of the cytotoxicity of aluminium oxide nanoparticles on selected mammalian cells. Toxicology in Vitro. 2011;25(8):1694-1700

[57] Sadiq R, Mahmood Khan Q, Mobeen A, Hashmat AJ. Vitrotoxicological assessment of iron oxide, aluminium oxide and copper nanoparticles in prokaryotic and eukaryotic cell types. Drug and Chemical Toxicology. 2014;38(2):152-161

[58] Braydich-Stolle L, Speshock J, Castle A, Smith M, Murdock R, Hussain S. Nanosized aluminum altered immune function. ACS Nano. 2010;4(7):3661-3670

[59] Lam C-W. Pulmonary toxicity of single-wall carbon nanotubes in mice 7 and 90 days after intratracheal instillation. Toxicological Sciences. 2003;77(1):126-134

[60] Li Y, Zhang X, Luo J, Huang W, Cheng J, Luo Z, Li T, Liu F, Xu G, Ke X, Li L, Geise H. Purification of CVD synthesized single-wall carbon nanotubes by different acid oxidation treatments. Nanotechnology. 2004;15(11):1645-1649

[61] Yazdan Madani S, Mandel A, Seifalian AM. A concise review of carbon nanotube's toxicology. Nano Reviews. 2013;4(1):21521

[62] Poland CA, Duffin R, Kinloch I, Maynard A, Wallace WAH, Seaton A, Stone V, Brown S, MacNee W, Donaldson K. Carbon nanotubes introduced into the abdominal cavity of mice show asbestos-like pathogenicity in a pilot study. Nature Nanotechnology. 2008; 3(7):423-428

[63] Xu J, Futakuchi M, Shimizu H, Alexander D, Yanagihara K, Fukamachi K, Suzui M, Kanno J, Hirose A, Ogata A, Sakamoto Y, Nakae D, Omori T, Tsuda H. Multi-walled carbon nanotubes translocate into the pleural cavity and induce visceral mesothelial proliferation in rats. Cancer Science. 2012;103(12):2045-2050

[64] Muller J, Huaux F, Moreau N, Misson P, Heilier J-F, Delos M, Arras M, Fonseca A, Nagy J, Lison D. Respiratory toxicity of multi-wall carbon nanotubes. Toxicology and Applied Pharmacology. 2005;207(3):221-231 
[65] Jastrzebska A-M, Kurtycz P, Olszyna A-R. Recent advances in graphene family materials toxicity investigations. Journal of Nanoparticle Research. 2012;14(12):1320

[66] Lalwani G, D'Agati M, Mahmud Khan A, Sitharaman B. Toxicology of graphene-based nanomaterials. Advanced Drug Delivery Reviews. 2016;105:109-144

[67] Seabra AB, Paula AJ, de Lima R, Alves OL, Durán N. Nanotoxicity of graphene and graphene oxide. Chemical Research in Toxicology. 2014;27(2):159-168

[68] Yang K, Li Y, Tan X, Peng R, Liu Z. Behavior and toxicity of graphene and its functionalized derivatives in biological systems. Small. 2012;9(9-10):1492-1503

[69] Zhang X, Yin J, Peng C, Hu W, Zhu Z, Li W, Fan C, Huang Q. Distribution and biocompatibility studies of graphene oxide in mice after intravenous administration. Carbon. 2011;49(3):986-995

[70] Singh SK, Singh MK, Kulkarni PP, Sonkar VK, Grácio JJA, Dash D. Amine-modified graphene: Thrombo-protective safer alternative to graphene oxide for biomedical applications. ACS Nano. 2012;6(3):2731-2740

[71] Singh SK, Singh MK, Nayak MK, Kumari S, Shrivastava S, Grácio JJA, Dash D. Thrombus inducing property of atomically thin graphene oxide sheets. ACS Nano. 2011;5(6):4987-4996

[72] Bengtson S, Knudsen KB, Kyjovska ZO, Berthing T, Skaug V, Levin M, Koponen IK, Shivayogimath A, Booth TJ, Alonso B, Pesquera A, Zurutuza A, Thomsen BL, Troelsen JT, Jacobsen NR, Vogel U. Differences in inflammation and acute phase response but similar genotoxicity in mice following pulmonary exposure to graphene oxide and reduced graphene oxide. Plos One. 2017;12(6):e0178355

[73] Ivask A, Voelcker NH, Seabrook SA, Hor M, Kirby JK, Fenech M, Davis TP, Ke PC. DNA melting and genotoxicity induced by silver nanoparticles and graphene. Chemical Research in Toxicology. 2015;28(5):1023-1035

[74] Pietroiusti A, Stockmann-Juvala H, Lucaroni F, Savolainen K. Nanomaterial exposure, toxicity, and impact on human health. Nanomedicine and Nanobiotechnology, Wiley-Blackwell. 2018;65(2):171-173, e1513

[75] Bouillard J, Vignes A. Nano-evaluris: An inhalation and explosion risk evaluation method for nanoparticle use. Part I: Description of the methodology. Journal of Nanoparticle Research. 2014;16:2149. https://doi.org/10.1007/s11051-013-2149-5

[76] Hristozov D, Malsch I. Hazards and risks of engineered nanoparticles for the environment and human health. Sustainability. 2009;1(4):1161-1194

[77] van Broekhuizen P, van Broekhuizen F, Cornelissen R, Reijnders L. Workplace exposure to nanoparticles and the application of provisional nanoreference values in times of uncertain risks. Journal of Nanoparticle Research. 2012;14(4):1-25

[78] De Volder M, Tawfick S, Baughman R, Hart J. Carbon nanotubes: Present and future commercial applications. Science (New York N.Y.). 2013;339:535-539 
[79] FrÃúhlich E. Cellular targets and mechanisms in the cytotoxic action of non-biodegradable engineered nanoparticles. Current Drug Metabolism. 2013;14:976-988

[80] ANSES. Development of a Specific Control Banding Tool for Nanomaterials. France: ANSES; 2010

[81] Paik S, Zalk D, Swuste P. Application of a pilot control banding tool for risk level assessment and control of nanoparticle exposures. The Annals of Occupational Hygiene. 2008; 52:419-428

[82] ISO. Nanotechnologies - Occupational Risk Management Applied to Engineered Nanomaterials. Part 2: Use of the Control Banding Approach. ISO/TS12901-2. Switzerland: ISO; 2014

[83] Hristozov D, Gottardo S, Semenzin E, Oomen A, Bos P, Peijnenburg W, van Tongeren M, Nowack B, Hunt N, Brunelli A, Scott-Fordsmand J, Tran L, Marcomini A. Frameworks and tools for risk assessment of manufactured nanomaterials. Environment International. 2016;95:36-53

[84] TNO. Stoffenmanager Nano: Description of the conceptual control banding model. Zeist, The Netherlands: TNO; 2011

[85] Jensen KA, Saber AT, Kristensen HV, Liguori B, Jensen AC, Koponen IK, Wallin H. Nanosafer Vs. 1.1 Nanomaterial Risk Assessment Using First Order Modeling. Poster session presented at Topical Scientific Workshop on Regulatory Challenges in Risk Assessment of Nanomaterials. Finland: Helsinki; 2014

[86] Prodanov D. Management of health risks related to the use of nanomaterials. An analogy with biosafety. Biomedical Reviews. 2018;28:104-109

[87] Afshari A, Matson U, Ekberg LE. Characterization of indoor sources of fine and ultrafine particles: A study conducted in a full-scale chamber. Indoor Air. 2005;15:141-150

[88] Zalk DM, Nelson DI. History and evolution of control banding: A review. Journal of Occupational and Environmental Hygiene. 2008;5(5):330-346

[89] Brouwer D, van Duuren-Stuurman B, Berges M, Jankowska E, Bard D, Mark D. From workplace air measurement results toward estimates of exposure? Development of a strategy to assess exposure to manufactured nano-objects. Journal of Nanoparticle Research. 2009;11(8):1867-1881

[90] Li Y, Li P, Yu H, Bian Y. Recent advances (2010-2015) in studies of cerium oxide nanoparticles' health effects. Environmental Toxicology and Pharmacology. 2016;44:25-29

[91] Belde P, Maillet J-C, Moclair F, Morelli A, Prodanov D, Roquet P. A list of associated tasks, activities and operations where exposure might occur. 2016. DOI: 10.5281/zenodo.804442 
